\title{
Message-passing theory for cooperative epidemics
}

\author{
Byungjoon Min ${ }^{1, a)}$ and Claudio Castellano 2, b) \\ 1) Department of Physics, Chungbuk National University, Cheongju, Chungbuk 28644, \\ Republic of Korea \\ ${ }^{2)}$ Istituto dei Sistemi Complessi (ISC-CNR), Via dei Taurini 19, I-00185 Roma, Italy
}

(Dated: February 25, 2020)

\begin{abstract}
The interaction among spreading processes on a complex network is a nontrivial phenomenon of great importance. It has recently been realized that cooperative effects among infective diseases can give rise to qualitative changes in the phenomenology of epidemic spreading, leading for instance to abrupt transitions and hysteresis. Here we consider a simple model for two interacting pathogens on a network and we study it by using the message-passing approach. In this way we are able to provide detailed predictions for the behavior of the model in the whole phase-diagram for any given network structure. Numerical simulations on synthetic networks (both homogeneous and heterogeneous) confirm the great accuracy of the theoretical results. We finally consider the issue of identifying the nodes where it is better to seed the infection in order to maximize the probability of observing an extensive outbreak. The message-passing approach provides an accurate solution also for this problem.
\end{abstract}

\begin{abstract}
How to predict and control mutually cooperative epidemics on a networked system is an outstanding problem of much interest. Previous attempts in this problem have assumed well-mixed populations and focused on the meanfield analysis. A full analysis of the cooperativity in epidemics at the level of single node is still lacking. In this work, we provide a message-passing approach in order to fully analyze cooperative epidemics on complex networks. We confirm the great accuracy of our theory with numerical simulations for both homogeneous and heterogeneous network structures. In addition, we reveal how to identify precisely influential spreaders in cooperative epidemics by using the message-passing equations.
\end{abstract}

\section{INTRODUCTION}

Many types of spreading phenomena, such as the propagation of infectious diseases or the diffusion of information or fads, are often strongly influenced by cooperativity effects. For instance, the epidemic spreading of a contagious disease can be dramatically boosted by the interaction with other infective pathogens. This cooperative effect is at the origin of devastating epidemics such as the co-occurrence of Spanish flu and pneumonia in $1918^{1 / 2}$ and the concurrent outbreaks of HIV/AIDS and a host of other diseases such as tuberculosis 3 . While the theoretical study of the epidemic spreading of a single-pathogen on complex topologies has a long and successful history 5 , intertwined with the theory of percolation and dynamical processes on networks 6 , the spreading of cooperative epidemics started to attract attention only recently.

Previous research has shown that cooperativity can give rise to violent outbreaks, which are the signature of an abrupt transition between the disease-free and the epidemic phase ${ }^{8} 12$. In addition to the discontinuous change, cooperative epidemic

\footnotetext{
a)Electronic mail: min.byungjoon@gmail.com

b)Electronic mail: claudio.castellano@roma1.infn.it
}

models are often characterized by the so-called "hybrid phase transition", a non-trivial critical phenomenon exhibiting features of both first- and second-order transitions 13 . The initial investigations on cooperative epidemics have been extended to analyze the role of degree heterogeneity 17 , clustering $18[19$, multiplex networks 20,22 , temporal networks 23 , cooperative recurrent epidemics 24 , more than two cooperating pathogens 25 .

In this work, we study the spreading of cooperative epidemics by extending to the case of two pathogens the message-passing approach fruitfully used for single-pathogen dynamics 26 . In order to account for the cooperativity between two different pathogens, we assume the probability of infection to depend on the state of the nodes. Specifically, when a node has already been infected with one of the pathogens, the probability that the second disease is transmitted to its neighbors through a contact grows. Previous investigations of this problem have been based on homogeneous and heterogeneous mean-field analyses. By suitably defining the transmission probabilities of the first and of the second disease, it is possible to treat the incoming transmission probability for each node as uncorrelated and in this way to extend the conventional message-passing approach to cooperative epidemics. Within the approach, we obtain a set of coupled equations whose numerical solution allows us to fully predict and control the effect of cooperativity at the level of single node, beyond well-mixed populations. Next, by using the framework we derive a number of analytical predictions for random network ensembles. Finally, as an important application, we tackle the problem of identifying influential spreaders, i.e., where an epidemic should be seeded to maximize the probability and the size of a global epidemic outbreak 1928 - 31 . The message-passing approach allows us to precisely predict these quantities for any pair of singly-infected seeds. Numerical simulations are performed to check all our analytical predictions on synthetic networks, finding overall an excellent agreement. 


\section{MODEL FOR COOPERATIVE EPIDEMICS}

The basic model for coinfections has been introduced by Chen, Ghanbarnejad, Cai and Grassberger (CGCG) ${ }^{8}$. It considers two different pathogens, $\mathrm{A}$ and $\mathrm{B}$, each evolving according to a SIR dynamics. Each node can be in one of three states with respect to each pathogen. For example, with respect to pathogen A a node can be susceptible $(S)$, infected and able to spread the infection further $(A)$ or recovered $(a)$. The state of a node is specified by indicating in which of the three possible states the node is with respect to each of the two pathogens. There are overall 9 possible individual states: $S, A, B, A B, A b, a B, a, b, a b$. Notice that states denoted by a single letter assume the omitted letter to be $S$.

In this paper, we propose a slightly different cooperative epidemic dynamics from the CGCG model. We still consider two different pathogens and in total 9 possible individual states. The cooperativity between two pathogens is defined as follows: for an agent infected with both pathogens, the probability to transmit the infection through a contact increases. The biological rationale of this modeling is that an infection with a pathogen may cause an individual to pass from the latent state to a fully infective one with respect to another pathogen. For instance, the infection of HIV is the most important risk factor for progressing from latent tuberculosis, which is hardly infectious, to active tuberculosis 4 . In this way the outgoing infection probability of the second disease increases when a node has already been infected with the other disease. On the other hand, in the CGCG model an individual infected with one disease has an increased probability to get infected with the second disease.

The crucial advantage of our modified cooperative model from a theoretical perspective is that the incoming transmission probabilities become effectively uncorrelated. The incoming probability does not depend on the current state of the node that can be infected, rather from the state of the node that attempts to infect. This lack of correlation allows us to use a message-passing approach to predict analytically the steady state behavior of cooperative epidemics. In this way it is possible to analyze and predict the cooperative epidemics on structured networks beyond the mean-field approximation 25 . As it will be shown below, this dynamics exhibits a behavior perfectly analogous to the original CGCG model. The original CGCG dynamics is actually not suitable for a messagepassing approach because incoming transmission probabilities are correlated. In that dynamics the incoming transmission probability is not only determined by the state of the source node but strongly depends on the state of the target node. Hence, the incoming transmission probabilities for each node are correlated and the message-passing approach cannot be applied to the CGCG dynamics.

In practice, the dynamics proceeds as follows. At the beginning of each discrete time step, a list of all infected nodes is recorded. Then we select at random from the list an infected node $i$ and one of its neighbors, $j$. If $i$ is infected with a single-pathogen A (or B) and it is susceptible with respect to the other, it attempts to infect $j$ with the pathogen and the infection is actually transmitted with probability $p_{A}$ (or $p_{B}$ ). If $i$ is not susceptible with respect to the other pathogen (i.e. it is currently infected or it has been infected in the past and is now recovered) then the probability of transmission is $q_{A}$ (or $\left.q_{B}\right)$. For a node in state $A B$, it is also possible that during the same time step $i$ infects $j$ first with pathogen A (with probability $p_{A}$ ) and then with pathogen $\mathrm{B}$ (probability $q_{B}$ ) or vice versa. After this infection attempt has been repeated for all the nodes in the list of infected nodes, all of them recover and the time step is over. In the rest of the paper we will consider, as in almost all previous studies, only the symmetric case, where $p_{A}=p_{B}=p$ and $q_{A}=q_{B}=q$. The investigation of the nonsymmetric case is an interesting issue that remains open for future work. In summary, the state of node $j$ is updated according to the following transition rules:

$$
\begin{gathered}
A+S \stackrel{p}{\longrightarrow} A+A \\
A+B \stackrel{p}{\longrightarrow} A+A B \\
A b+S \stackrel{q}{\longrightarrow} A b+A \\
A b+B \stackrel{q}{\longrightarrow} A b+A B \\
B+S \stackrel{p}{\longrightarrow} B+B \\
B+A \stackrel{p}{\longrightarrow} B+A B \\
a B+S \stackrel{q}{\longrightarrow} a B+B \\
a B+A \stackrel{q}{\longrightarrow} a B+A B \\
A B+S \stackrel{p q}{\longrightarrow} A B+A B \\
A B+S \stackrel{\frac{1}{2} p(1-q)}{\longrightarrow} A B+A \\
A B+S \stackrel{\frac{1}{2} p(1-q)}{\longrightarrow} A B+B \\
A \stackrel{1}{\longrightarrow} a \\
B \stackrel{1}{\longrightarrow} a \\
A B \stackrel{1}{\longrightarrow} a \\
A B \stackrel{1}{\longrightarrow} A b
\end{gathered}
$$

\section{MESSAGE-PASSING THEORY}

\section{A. Message-passing equations}

In the symmetric case, by construction the probabilities of the process do not depend on which of the two pathogens is considered. We can then define a single function $u_{i j}$, as the probability that, at the end of the dynamics, node $i$ has not been infected by node $j$ with a given pathogen. In analogy with the case of SIR single-pathogen dynamics $28 \mid 31$, the message-passing equations for these quantities are 


$$
1-u_{i j}=\frac{1}{2}(p+q)\left(1-\prod_{k \in \partial j \backslash i} u_{j k}\right)\left(1-\prod_{k \in \partial j} u_{j k}\right)+p\left(1-\prod_{k \in \partial j \backslash i} u_{j k}\right)\left(\prod_{k \in \partial j} u_{j k}\right) .
$$

where $k \in \partial j$ represents the set of neighbors of node $j$ and $k \in \partial j \backslash i$ is the set of neighbors of node $j$ excluding node $i$. The second term on the r.h.s. takes into account the case node $i$ is infected with a pathogen by a node $j$ that is never infected with the other pathogen. The first term accounts instead for the case $i$ is infected with a pathogen by a node $j$ that at the end is also infected with the other. The factor $1 / 2$ in $(p+q) / 2$ is the probability that the node is infected with the given pathogen earlier or later than with the other. Here we assume that the networks have a locally tree-like structure, i.e., they contain no short loops. For highly clustered networks, Eq. 1 cannot guarantee to produce accurate probabilities and hence more advanced treatments $32 \sqrt{34}$ are needed. In the noncooperating case, $q=p$, Eq. (1) correctly coincides with the message-passing equation for single-pathogen SIR dynamics. Note that $u_{i j}=1$ is the trivial fixed point corresponding to no outbreak propagation.

\section{B. Derivation of the epidemic threshold}

In order to find the epidemic threshold, let us set $u_{i j}=1-$ $\varepsilon_{i j}$ and expand Eq. (1) for small $\varepsilon_{i j}$ :

$$
\begin{aligned}
\varepsilon_{i j} & =\frac{1}{2}(p+q)\left[1-\prod_{k \in \partial j \backslash i}\left(1-\varepsilon_{j k}\right)\right]\left[1-\prod_{k \in \partial j}\left(1-\varepsilon_{j k}\right)\right]+p\left[1-\prod_{k \in \partial j \backslash i}\left(1-\varepsilon_{j k}\right)\right]\left[\prod_{k \in \partial j}\left(1-\varepsilon_{j k}\right)\right] \\
& \approx \frac{1}{2}(p+q) \sum_{k \in \partial j \backslash i} \varepsilon_{j k} \sum_{k \in \partial j} \varepsilon_{j k}+p\left(\sum_{k \in \partial j \backslash i} \varepsilon_{j k}-\frac{1}{2} \sum_{\substack{k, l \in \partial j \backslash i \\
l \neq k}} \varepsilon_{j k} \varepsilon_{j l}\right)\left(1-\sum_{k \in \partial j} \varepsilon_{j k}\right) .
\end{aligned}
$$

Neglecting second and higher order terms, we obtain

$$
\varepsilon_{i j} \approx p \sum_{k \in \partial j \backslash i} \varepsilon_{j k}
$$

Defining the $2 E \times 2 E$ non-backtracking matrix $B^{35 / 36}$ (where $E$ is the number of edges in the network) with elements

$$
B_{i \rightarrow j, l \rightarrow k}=\delta_{j l}\left(1-\delta_{i k}\right)
$$

we can write

$$
\varepsilon=p B \varepsilon .
$$

An immediate consequence is that the epidemic threshold is given by the inverse of the principal eigenvalue $\Lambda_{M}(B)$ of the non-backtracking matrix $B$

$$
p_{c}=\frac{1}{\Lambda_{M}(B)} \text {. }
$$

The parameter $q$ does not appear in Eq. (6), hence $p_{c}$ coincides with the epidemic threshold for single-pathogen dynamics.

Keeping terms up to second order, we obtain

$$
\varepsilon_{i j} \approx p \sum_{k \in \partial j \backslash i} \varepsilon_{j k}+\frac{1}{2}(q-p) \sum_{k \in \partial j \backslash i} \varepsilon_{j k} \sum_{k \in \partial j} \varepsilon_{j k}-\frac{p}{2} \sum_{\substack{k, l \in \partial j \backslash i \\ l \neq k}} \varepsilon_{j k} \varepsilon_{j l} .
$$

Depending on $q$, the second order terms in Eq. (7) can be either positive or negative. The transition of the outbreak size at $p_{c}$ becomes discontinuous when $q$ becomes larger than a certain threshold $q_{c}$, while the transition remains continuous when $q<q_{c}$. Note that the ordinary SIR model always produces a continuous transition since the second order term for the ordinary $\operatorname{SIR}$ model $(q=p)$ is always negative.

\section{Outbreak size}

Once the coupled equations (1) are solved - for example, by iteration - the probabilities that node $i$ has been infected with both pathogens, or with one pathogen but not with the other are, respectively:

$$
\begin{aligned}
& \rho_{i}^{a b}=\left(1-\prod_{j \in \partial i} u_{i j}\right)^{2} \\
& \rho_{i}^{a}=\left(1-\prod_{j \in \partial i} u_{i j}\right)\left(\prod_{j \in \partial i} u_{i j}\right) .
\end{aligned}
$$


The average size of cooperative epidemic outbreaks is therefore

$$
\rho_{a b}=\frac{1}{N} \sum_{i}\left(1-\prod_{j \in \partial i} u_{i j}\right)^{2} .
$$

By the same token, the fraction of nodes infected only by a given pathogen is

$$
\rho_{a}=\frac{1}{N} \sum_{i}\left(1-\prod_{j \in \partial i} u_{i j}\right)\left(\prod_{j \in \partial i} u_{i j}\right) .
$$

\section{Probability of coinfection outbreaks}

Let us determine the probability of coinfection outbreaks initiated by two singly-infected seed nodes. For instance, pathogen $\mathrm{A}$ is initially seeded in node $i$ while pathogen $\mathrm{B}$ is seeded in node $j$. A necessary condition for the formation of a global coinfection is that each pathogen is individually able to originate an extensive single-pathogen outbreak, so that the two outbreaks can both infect some nodes. Only after independently evolving single-pathogen outbreaks have started to overlap, cooperativity may begin to play its role. To assess the probability of a single-pathogen outbreak, we can use ordinary message-passing equations for usual single-pathogen $\operatorname{SIR}^{2628}$, that is

$$
v_{i j}=1-p\left(1-\prod_{k \in \partial j \backslash i} v_{j k}\right) .
$$

Then the probability of observing a coinfection outbreak initiated by two singly-infected seeds, $i$ and $j$, is

$$
P_{a b}^{i j}=\left(1-\prod_{k \in \partial i} v_{i k}\right)\left(1-\prod_{l \in \partial j} v_{j l}\right) .
$$

The average probability for every pair of seeds $i$ and $j$ is then

$$
P_{a b}=\frac{1}{\left(\begin{array}{c}
N \\
2
\end{array}\right)} \sum_{i, j}\left(1-\prod_{k \in \partial i} v_{i k}\right)\left(1-\prod_{l \in \partial j} v_{j l}\right) .
$$

Notice that, since it is based on single-pathogen quantities, $P_{a b}$ changes continuously at the transition and has no dependence on $q$ whatsoever.

\section{ON RANDOM NETWORKS}

The equations presented in the previous section can be easily solved numerically for any given network, thus providing predictions for all observable of interest. In order to obtain fully analytical predictions it is possible to perform a further step, assuming that all variables $u_{i j}$ share the same value $u$ and the network structure is given by the degree distribution $P(k)$, so that Equation (1) can be written as

$$
\begin{aligned}
1-u & =\sum_{k=1}^{\infty} \frac{k P(k)}{\langle k\rangle}\left[\frac{1}{2}(p+q)\left(1-u^{k-1}\right)\left(1-u^{k}\right)\right. \\
& \left.+p\left(1-u^{k-1}\right) u^{k}\right]
\end{aligned}
$$

In the same way the expressions for the outbreak size $\rho_{a b}$ and $\rho_{a}$ become

$$
\begin{aligned}
\rho_{a b} & =\sum_{k} P(k)\left(1-u^{k}\right)^{2} . \\
\rho_{a} & =\sum_{k} P(k)\left(1-u^{k}\right) u^{k},
\end{aligned}
$$

while

$$
P_{a b}=\sum_{k} P(k)\left(1-v^{k}\right)^{2} .
$$

where $v$ is the solution of Eq. (15) for $q=p$.

Defining the function

$$
\begin{aligned}
f(u) & =1-u-\sum_{k=1}^{\infty} \frac{k P(k)}{\langle k\rangle} \\
& \times\left[\frac{1}{2}(p+q)\left(1-u^{k-1}-u^{k}+u^{2 k-1}\right)+p\left(u^{k}-u^{2 k-1}\right)\right]
\end{aligned}
$$

the epidemic threshold is determined by the condition $f^{\prime}(1)=$ 0, i.e.,

$$
p \sum_{k=1}^{\infty} \frac{k(k-1) P(k)}{\langle k\rangle}=1 .
$$

Thus, the epidemic threshold is the same as the ordinary SIR model as

$$
p_{c}=\frac{\langle k\rangle}{\left\langle k^{2}\right\rangle-\langle k\rangle} .
$$

The point where the transition becomes discontinuous can be identified by the conditions $f^{\prime}(1)=0$ and $f^{\prime \prime}(1)=0$. Since

$$
f^{\prime \prime}(1)=\frac{1}{\langle k\rangle}\left[(2 p-q)\left\langle k^{3}\right\rangle-(4 p-q)\left\langle k^{2}\right\rangle+2 p\langle k\rangle\right] .
$$

the change from continuous to hybrid transition occurs for $w=q / p>w_{c}$ where

$$
w_{c}=\frac{2\left\langle k^{3}\right\rangle-4\left\langle k^{2}\right\rangle+2\langle k\rangle}{\left\langle k^{3}\right\rangle-\left\langle k^{2}\right\rangle} .
$$

\section{A. Erdős-Rényi networks}

For Erdős-Rényi (ER) networks with average degree $\langle k\rangle=$ $z$, Eqs. 20, and 22 yield

$$
p_{c}=\frac{1}{z}, \quad w_{c}=\frac{2(1+z)}{2+z} .
$$




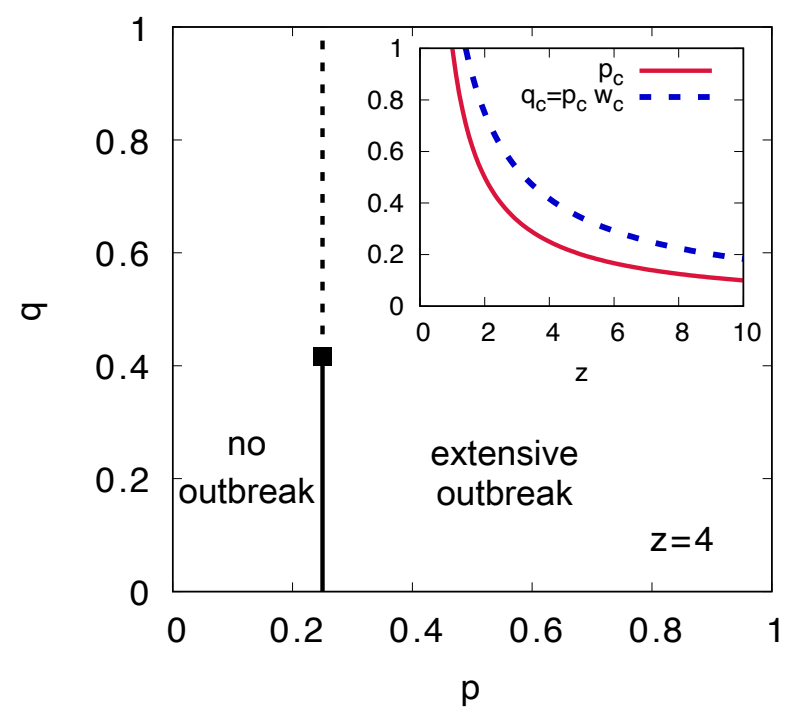

Figure 1. Phase diagram for ER networks obtained by the theory. The solid and dashed lines respectively represent continuous and discontinuous transitions. The tricritical point $q_{c}$ where a continuous transition becomes discontinuous is represented by a filled symbol. (inset) Dependence on the average degree $z$ of the critical values $p_{c}$ and $q_{c}=p_{c} w_{c}$.

In Fig. 1. we display, for fixed $z=4$, the phase-diagram of the model. In the inset of Fig. 1, we plot the two critical values $p_{c}$ and $q_{c}=p_{c} w_{c}$ as a function of the average connectivity $z$. While $p_{c}$ separates regions with and without extensive coinfection outbreaks, $q_{c}=p_{c} w_{c}$ discriminates when the transition is continuous (for $q<q_{c}$ ) and when it is discontinuous (for $q>q_{c}$ ).

For ER networks we can determine analytically the behavior of relevant observables for any value of $p$ and $q$. Poisson degree distribution implies that $\sum_{k} P(k) u^{k}=$ $z^{-1} \sum_{k} k P(k) u^{k-1}=e^{z(u-1)}$, so that the equation for the probability $u$ is

$$
\begin{aligned}
1-u & =\frac{1}{2}(p+q)\left[1-e^{z(u-1)}-u e^{z(u-1)}+u e^{z\left(u^{2}-1\right)}\right] \\
& +p\left[u e^{z(u-1)}-u e^{z\left(u^{2}-1\right)}\right]
\end{aligned}
$$

the equation for the outbreak size is

$$
\rho_{a b}=1-2 e^{z(u-1)}+e^{z\left(u^{2}-1\right)}
$$

while

$$
\rho_{a}=e^{z(u-1)}-e^{z\left(u^{2}-1\right)},
$$

and the probability to have a coinfection outbreak is

$$
P_{a b}=1-2 e^{z(v-1)}+e^{z\left(v^{2}-1\right)}
$$

where $v$ is the solution of

$$
1-v=p\left[1-e^{z(v-1)}\right]
$$

\section{B. Power-law distributed networks}

For power-law distributed networks with degree distribution $P(k)=(\gamma-1) m^{\gamma-1} k^{-\gamma}$ (with $m$ the minimum degree) the prediction for the threshold Eq. (20) becomes

$$
p_{c}=\frac{1}{m \frac{\gamma-2}{\gamma-3}-1} \text {. }
$$

Replacing the expressions for the moments of the distribution $P(k)$ in Eq. 22) it turns out that the critical value $w_{c}$ increases as $\gamma$ is lowered, up to a value $w_{c}=2$ for $\gamma=4$. For $\gamma<4$ the third moment of the degree distribution diverges and therefore, in the infinite size limit Eq. 22) predicts $w_{c}=2$.

\section{NUMERICAL SIMULATIONS}

We compare the analytical results obtained above with direct numerical simulations of the coinfective spreading dynamics. As contact patterns for the epidemic process we consider either homogeneous ER networks or power-law distributed networks built according to the configuration mode ${ }^{6}$. Numerically, we consider an outbreak to be epidemic if the final density of coinfected nodes $\rho_{a b}$ is larger than a threshold equal to 0.001 . We have checked that results do not change if different values are considered.

\section{A. Erdős-Rényi networks}

In Fig. 2 we present a comparison between the theoretical predictions of the message-passing approach, complemented with the random networks with a given degree distribution and numerical simulations performed on ER networks. The agreement between theory and simulations is excellent overall. In agreement with Eq. 23, the transition point $p_{c}$ does not depend on $w=q / p$. The coinfection outbreak size $\rho_{a b}$ and the fraction $\rho_{a}$ of singly-infected nodes change continuously or discontinuously at the threshold depending on whether $w$ is smaller or larger than $w_{c}$. Conversely, the probability of epidemic outbreaks always undergoes a continuous transition and is completely independent from $q$.

Further insight into the nature of the transition is provided in Fig. 3 For different values of $w$ the distribution of avalanche sizes at the critical point decays as a power-law with the same exponent $\tau=3 / 2$ valid for single-pathogen SIR dynamics. Above the critical value $w_{c}$, however, the hybrid nature of the transition results in the formation of a peak at a finite value of $\rho_{a b}$.

\section{B. Power-law distributed networks}

In Fig. 4 we compare the theoretical predictions of the message-passing approach and numerical results obtained on power-law distributed networks. In this example, we generate a network with degree exponent $\gamma=3.5$ according to the 

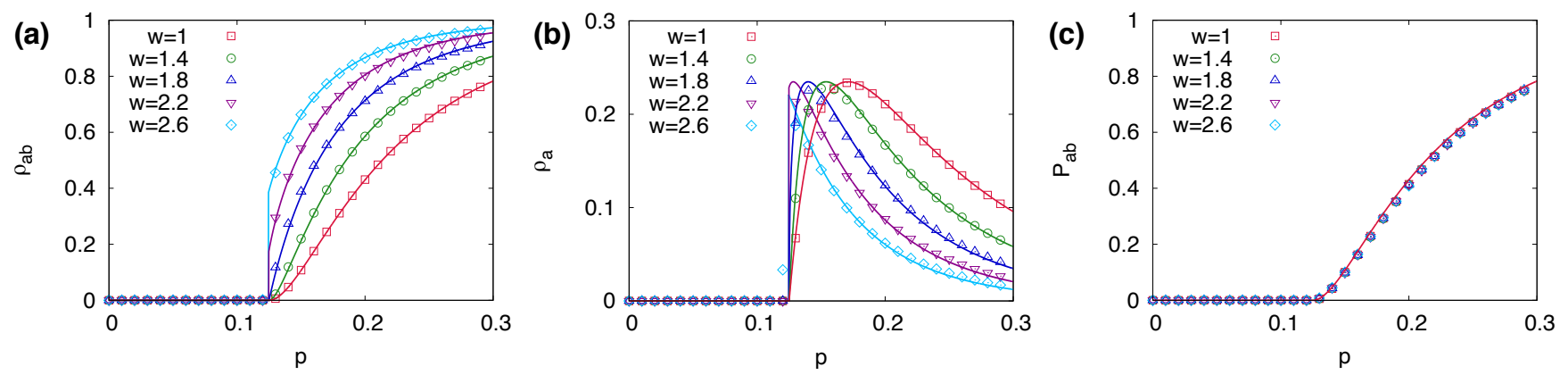

Figure 2. (a) Values of $\rho_{a b}$, (b) $\rho_{a}$ and of (c) the probability $P_{a b}$ of coinfection epidemic outbreaks on ER networks with $N=10^{5}$ and $\langle k\rangle=8$. For this choice of parameters, the theory predicts that $p_{c}=1 / 8$ and $w_{c}=1.8$. Numerical results (symbols) are obtained by averaging over $10^{5}$ realizations. Theoretical results (continuous lines) are obtained by means of Eqs. 25, 26] and [27, respectively.

configuration model 6 . The network with $\gamma=3.5$ is heterogeneous but still maintains a non-zero epidemic threshold in the thermodynamic limit. Also in this case the transition point depends only on $p$ and not on $q$, in agreement with Eq. (6). What changes when $q$ increases is the nature of the transition. Above a critical value $w=w_{c}$ for the ratio $w=q / p$ the transition becomes hybrid. The coinfection outbreak size $\rho_{a b}$ and $\rho_{a}$ both jump discontinuously at the threshold, while the probability $P_{a b}$ of epidemic outbreaks always undergoes a continuous transition and is completely independent from $q$. Numerical results agree well with this picture. There is a small discrepancy in $\rho_{a b}$ and $\rho_{a}$ below $p_{c}$ due to finite size effects. $\rho_{a b}$ and $\rho_{a}$ are average values calculated over realizations such that $\rho_{a b}>0.001$. Even if the probability of observing extensive outbreaks is zero below the threshold in the thermodynamic limit, for finite system and sufficiently many realizations there is still a little, yet very low, chance to reach the upper branch of the two stable solutions in the bistable region. The values different from zero in Fig. 4 immediately below the threshold are the effect of these spurious events.

\section{FINDING INFLUENTIAL SPREADERS}

We now turn to the identification of influential spreaders in the network, a problem which has attracted a large in-
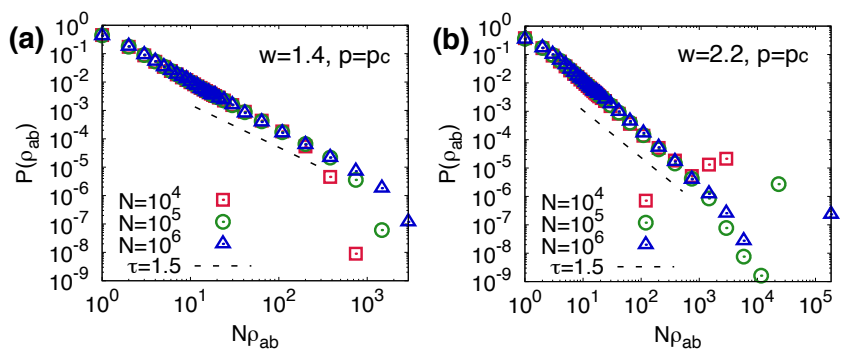

Figure 3. The size distribution of $\rho_{a b}$ at (a) $w=1.4$ and (b) $w=2.2$ and $p=p_{c}=0.125$ for ER networks with $N=10^{4}, 10^{5}, 10^{6}$ and $\langle k\rangle=8$. terest recently 29/30. In practice we are interested in predicting, given two specific nodes $i$ and $j$ as seeds (each infected with a single-pathogen), what is the probability that an extensive coinfection outbreak occurs and what is its expected size. Within the message-passing approach, the quantity $P_{a b}^{i j}$ calculated in Eq. (13) is the answer to the first question, while the size of the outbreak is given by Eq. (10).

In Fig. 5] we compare this analytical prediction to numerical results for an ER and a SF network with the two initiators randomly chosen. The message-passing approach allows us to determine with great accuracy the ability of a pair of nodes to generate global coinfection epidemic [see panels $(\mathrm{a}, \mathrm{b}, \mathrm{e}, \mathrm{f})$ of Fig. 5]. Such ability depends strongly on $p$ and on which pair of seeds is selected, while it is independent of the value of $q$, as predicted by Eq. (13). Once an outbreak develops its size is instead insensitive to the location of the initial seeds [see panels (c,d,g,h) of Fig. 5], while it changes depending on the value of the cooperativity parameter $w$. This is again in excellent agreement with the theory.

Eq. (13) provides an analytical expression for the probability $P_{a b}^{i j}$ of observing an extensive cooperative outbreak originated from nodes $i$ and $j$

$$
P_{a b}^{i j}=\left(1-v^{k_{i}}\right)\left(1-v^{k_{j}}\right) .
$$

In Fig. 6 we test numerically this prediction finding again a remarkably good agreement with relatively small computational cost. In the vicinity of the critical point it is possible to set $v=1-\varepsilon$ and expand Eq. 30 as a function of $\varepsilon$, obtaining

$$
P_{a b}^{i j} \sim \varepsilon^{2} k_{i} k_{j} .
$$

This result connects the spreading influence of a pair of nodes with their degree centrality. In the case a single doublyinfected seed is considered Eq. (31) predicts a dependence on the square of the seed degree, in agreement with recent numerical results 19 .

\section{CONCLUSIONS}

In this paper, we propose a model of mutually cooperative coinfections with uncorrelated incoming transmission proba- 

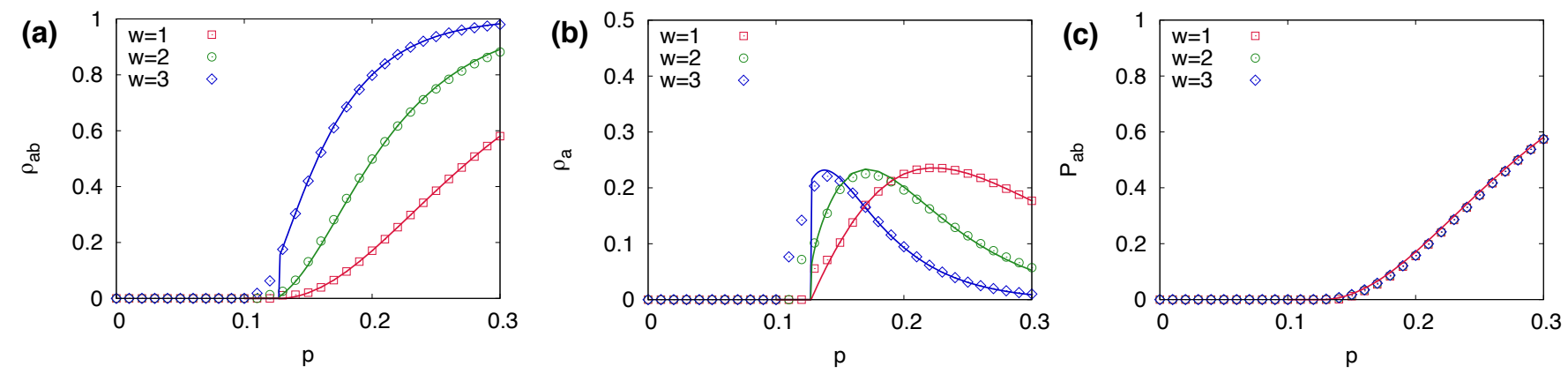

Figure 4. (a) Values of $\rho_{a b}$, (b) $\rho_{a}$ and (c) the probability $P_{a b}$ of coinfection epidemic outbreaks on a power-law distributed network with $N=10^{4}$ and $\gamma=3.5$. Numerical results (symbols) are obtained by averaging over $10^{5}$ realizations. Theoretical results (continuous lines) are obtained by means of Eqs. [10, , 11] and (14), respectively.
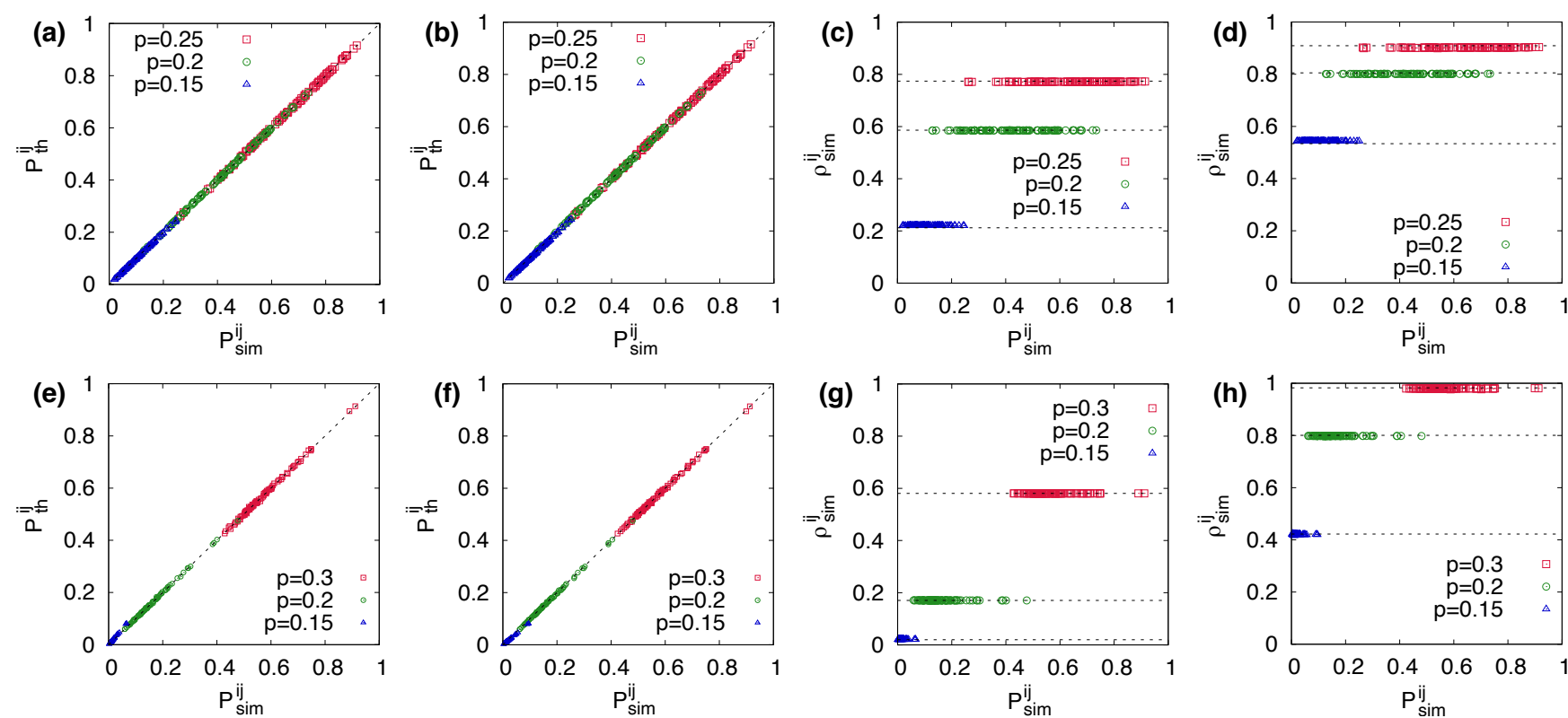

Figure 5. Comparison between the message-passing theory and numerical results for the probability of epidemic outbreaks for two singlyinfected seeds when (a) $w=1.4$ and (b) $w=2.2$ on an ER network with $\langle k\rangle=8$ and size $N=10^{4}$ and when (e) $w=1$ and (f) $w=3$ on a SF network with $\gamma=3.5$ and size $N=10^{4}$. Comparison between the message-passing theory (dashed lines) and numerical results (symbols) for the size of epidemic outbreaks generated by two singly-infected seeds with (c) $w=1.4$ (c) and (d) $w=2.2$ on an ER network and with (g) $w=1$ and (h) $w=3$ on a SF network.

bilities and study the behavior of the model based on messagepassing equations. In this way we are able to provide accurate predictions - beyond the mean-field approximation - of the phase-diagram, the probability of epidemic outbreaks, the size of epidemic outbreaks for any given network structure. We confirm our predictions with numerical simulations on random networks for both homogeneous and heterogeneous networks. By applying this theoretical framework we can calculate the spreading influence of individual nodes and thus identify which of them maximize the probability of a global extensive outbreak. Our study provides a systematic way to analyze the cooperativity in spreading processes on complex networks. Further studies will be needed to examine the case of nonsymmetric transmission probabilities, interacting epi- demics in clustered networks (for which the present messagepassing approach becomes inaccurate) or in meta-populations networks. Moreover, since most real-world contagion processes take place in multiple different layers of networks, a natural extension of our work would be the consideration of cooperative epidemics on a multilayer network. While epidemic spreading on multilayer networks has been studied extensively 37,39 , cooperative epidemics on multilayer networks have not been fully investigated. Our model and messagepassing theory can guide to the study of cooperative epidemics on multilayer networks. 

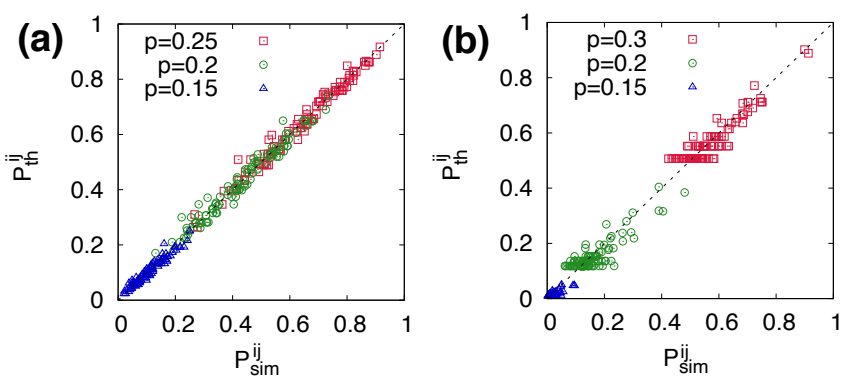

Figure 6. Comparison between the approximation (Eq. 30p) and numerical results for the probability of epidemic outbreaks for two singly-infected seeds when (a) $w=2.2$ on an ER network with $\langle k\rangle=$ 8 and size $N=10^{4}$ and (b) $w=3$ on a SF network with $\gamma=3.5$ and size $N=10^{4}$.

\section{ACKNOWLEDGMENTS}

We acknowledge financial support from the 2018 Short Term Mobility program of Consiglio Nazionale delle Ricerche (CNR) of Italy. This work was supported by the National Research Foundation of Korea (NRF) grant funded by the Korea government (MSIT) (No. 2018R1C1B5044202). We also thank C. Han for high-performance computing support.

\section{REFERENCES}

${ }^{1}$ D. M. Morens, J. K. Taubenberger, and A. S. Fauci, "Predominant role of bacterial pneumonia as a cause of death in pandemic influenza: Implications for pandemic influenza preparedness," The Journal of Infectious Diseases 198, 962 (2008)

${ }^{2}$ J. F. Brundage and G. Shanks, "Deaths from bacterial pneumonia during 1918-19 influenza pandemic," Emerg. Infect Dis. 14, 1193-1199 (2008)

${ }^{3}$ M. S. Sulkowski, "Viral hepatitis and HIV coinfection," Journal of Hepatology 48, 353-367 (2008)

${ }^{4}$ A. Pawlowski, M. Jansseon, M. Sköld, M. E. Rottenberg, and G. Källenius, "Tuberculosis and hiv co-infection," PloS Pathogens 8(2), e1002464 (2012)

${ }^{5}$ R. Pastor-Satorras, C. Castellano, P. Van Mieghem, and A. Vespignani, "Epidemic processes in complex networks," Rev. Mod. Phys. 87, 925-979 (2015)

${ }^{6}$ M. Newman, Networks: An Introduction (Oxford University Press, Inc., New York, NY, USA, 2010).

${ }^{7}$ S. N. Dorogovtsev, A. V. Goltsev, and J. F. F. Mendes, "Critical phenomena in complex networks," Rev. Mod. Phys. 80, 1275-1335 (2008)

${ }^{8}$ L. Chen, F. Ghanbarnejad, W. Cai, and P. Grassberger, "Outbreaks of coinfections: The critical role of cooperativity," EPL (Europhysics Letters) 104, 50001 (2013)

${ }^{9}$ W. Cai, L. Chen, F. Ghanbarnejad, and P. Grassberger, "Avalanche outbreaks emerging in cooperative contagions," Nature physics 11, 936-940 (2015)

${ }^{10} \mathrm{P}$. Grassberger, L. Chen, F. Ghanbarnejad, and W. Cai, "Phase transitions in cooperative coinfections: Simulation results for networks and lattices," Phys. Rev. E 93, 042316 (2016)

${ }^{11}$ H.-K. Janssen and O. Stenull, "First-order phase transitions in outbreaks of co-infectious diseases and the extended general epidemic process," EPL (Europhys. Lett.) 113 (2016), 10.1209/0295-5075/113/26005

${ }^{12}$ W. Choi, D. Lee, and B. Kahng, "Mixed-order phase transition in a twostep contagion model with a single infectious seed," Phys. Rev. E 95 (2017), 10.1103/PhysRevE.95.022304

${ }^{13}$ A. V. Goltsev, S. N. Dorogovtsev, and J. F. F. Mendes, " $k$," Phys. Rev. E 73, $056101(2006)$
${ }^{14}$ B. Min and M. San Miguel, "Competing contagion processes: complex contagion triggered by simple contagion," Scientific Reports 8, 10422 (2018)

${ }^{15}$ D. Lee, W. Choi, J. Kertész, and B. Kahng, "Universal mechanism for hybrid percolation transitions," Scientific Reports 7 (2017), 10.1038/s41598017-06182-3

${ }^{16}$ Y. Baek, K. Chung, M. Ha, H. Jeong, and D. Kim, "Role of hubs in the synergistic spread of behavior,' Phys. Rev. E 99, 020301 (2019)

${ }^{17}$ P.-B. Cui, F. Colaiori, and C. Castellano, "Mutually cooperative epidemics on power-law networks,"'Phys. Rev. E 96, 022301 (2017)

${ }^{18}$ L. Hébert-Dufresne and B. M. Althouse, "Complex dynamics of synergistic coinfections on realistically clustered networks," Proceedings of the National Academy of Sciences 112, 10551-10556 (2015) https://www.pnas.org/content/112/33/10551.full.pdf

${ }^{19}$ P.-B. Cui, F. Colaiori, and C. Castellano, "Effect of network clustering on mutually cooperative coinfections,' Phys. Rev. E 99, 022301 (2019)

${ }^{20}$ N. Azimi-Tafreshi, "Cooperative epidemics on multiplex networks," Phys. Rev. E 93, 042303 (2016)

${ }^{21}$ X. Wei, S. Chen, X. Wu, J. Feng, and J. an Lu, "A unified framework of interplay between two spreading processes in multiplex networks," EPL (Europhysics Letters) 114, 26006 (2016)

${ }^{22}$ Q.-H. Liu, W. Wang, S.-M. Cai, M. Tang, and Y.-C. Lai, "Synergistic interactions promote behavior spreading and alter phase transitions on multiplex networks," Phys. Rev. E 97, 022311 (2018)

${ }^{23}$ J. P. Rodríguez, F. Ghanbarnejad, and V. M. Eguíluz, "Risk of coinfection outbreaks in temporal networks: A case study of a hospital contact network,' Frontiers in Physics 5, 46 (2017)

${ }^{24}$ L. Chen, F. Ghanbarnejad, and D. Brockmann, "Fundamental properties of cooperative contagion processes," New Journal of Physics 19, 103041 (2017)

${ }^{25}$ F. Zarei, S. Moghimi-Araghi, and F. Ghanbarnejad, "Exact solution of generalized cooperative susceptible-infected-removed (sir) dynamics," Phys. Rev. E 100, 012307 (2019)

${ }^{26}$ B. Karrer and M. E. J. Newman, "Message passing approach for general epidemic models,"'Phys. Rev. E 82, 016101 (2010)

${ }^{27}$ A. Y. Lokhov, M. Mézard, H. Ohta, and L. Zdeborová, "Inferring the origin of an epidemic with a dynamic message-passing algorithm," Phys. Rev. E 90, 012801 (2014)

${ }^{28} \mathrm{~B}$. Min, "Identifying an influential spreader from a single seed in complex network s via a message-passing approach," The European Physical Journal B 91, 18 (2018)

${ }^{29}$ M. Kitsak, L. K. Gallos, S. Havlin, F. L iljeros, L. Muchnik, H. E. Stanley, and H. A. Makse, "Identification of influential spreaders in complex networks," Nature Physics 6, 888-893 (2010)

${ }^{30}$ L. Lu, D. Chen, X.-L. Ren, Q.-M. Zhang, Y.-C. Zhang, and T. Zhou, "Vital nodes identification in complex networks," Physics Reports 650, 1 - 63 (2016)

${ }^{31} \mathrm{~F}$. Radicchi and C. Castellano, "Leveraging percolation theory to single out influential spreaders in networks," Phys. Rev. E 93, 062314 (2016)

${ }^{32}$ M. E. J. Newman, "Random graphs with clustering," Phys. Rev. Lett. 103 (2009), 10.1103/PhysRevLett.103.058701

${ }^{33} \mathrm{~F}$. Radicchi and C. Castellano, "Beyond the locally treelike approximation for percolation on real networks," Phys. Rev. E 93 (2016), 10.1103/PhysRevE.93.030302

${ }^{34}$ G. T. Cantwell and M. E. J. Newman, "Message passing on networks with loops," Proceedings of the National Academy of Sciences 116, 2339823403 (2019) https://www.pnas.org/content/116/47/23398.full.pdf

${ }^{35} \mathrm{~K}$. Hashimoto, "Zeta functions of finite graphs and representations of p-adic g roups," Adv. Stud. Pure Math. 15, 211-280 (1989)

${ }^{36}$ B. Karrer, M. E. J. Newman, and L. Zdeborová, "Percolation on sparse networks,'Phys. Rev. Lett. 113, 208702 (2014)

${ }^{37}$ A. Saumell-Mendiola, M. Ángeles Serrano, and M. Boguñá, "Epidemic spreading on interconnected networks," Phys. Rev. E 86 (2012), 10.1103/PhysRevE.86.026106

${ }^{38}$ M. Kivelä, A. Arenas, M. Barthelemy, J. P. Gleeson, Y. Moreno, and M. A. Porter, "Multilayer networks," J. Compl. Netw. 2 (2014), 10.1093/comnet/cnu016

${ }^{39}$ B. Min, S.-H. Gwak, N. Lee, and K.-I. Goh, "Layer-switching cost and optimality in information spreading on multiplex networks," Sci. Rep. 6 (2016), 10.1038/srep21392 